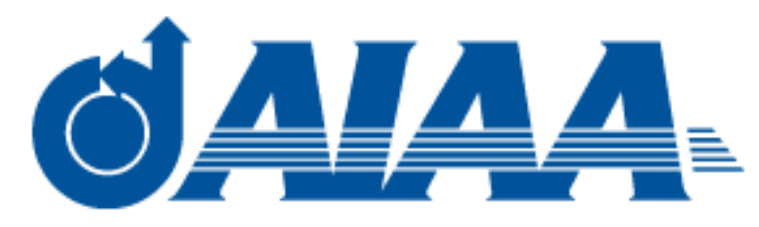

\title{
Scaling of Lift Coefficient of an Airfoil with Leading Edge Serrations
}

\begin{tabular}{|c|c|}
\hline Journal: & AIAA Journal \\
\hline Manuscript ID & 2018-12-J058168.R1 \\
\hline Manuscript Type: & Technical Note \\
\hline $\begin{array}{l}\text { Date Submitted by the } \\
\text { Author: }\end{array}$ & 23-Mar-2019 \\
\hline Complete List of Authors: & $\begin{array}{l}\text { Stalnov, Oksana; Technion Israel Institute of Technology, Faculty of } \\
\text { Aerospace Engineering } \\
\text { Chong, Tze Pei; Brunel University, School of Engineering and Design }\end{array}$ \\
\hline Subject Index Category: & 20100 Aeroacoustics < 20000 FLUID DYNAMICS \\
\hline $\begin{array}{r}\text { Select ONE Subject Index for } \\
\text { the Table of Contents. } \\
<\text { br }>\text { This is where your } \\
\text { paper will show up in the } \\
\text { Table of Contents: }\end{array}$ & 20000 FLUID DYNAMICS \\
\hline
\end{tabular}




\title{
Scaling of Lift Coefficient of an Airfoil with Leading Edge Serrations
}

\author{
Oksana Stalnov* \\ Technion - Israel Institute of Technology, Haifa, 76542601, Israel \\ Tze Pei Chong ${ }^{\dagger}$ \\ Brunel University London, Uxbridge, UB8 3PH, UK
}

\section{Introduction}

$\mathbf{I}^{\mathrm{N}}$ $\mathrm{N}$ most engineering applications the leading edge of a man made wing is straight. Nevertheless, this is not the case for natural flyers or swimmers. For example, owls have leading edge serrations on their wings and humpback whales have tubercles on their pectoral flippers. Owing to these morphological features owls can perform an extremely quiet flight [1] and humpback whales can achieve high agility [2, 3]. Utilizing these features during the pursuit of a prey owls can hunt in stealth, whilst the humpback whales can easily maneuver in the ocean water despite their great size and weight. To achieve better performance engineers and scientists are following the nature's footsteps by mimicking these morphological features.

Modifications to the leading edge geometry, in form of leading edge serrations, have shown to be acoustically beneficial when a wing is placed in a highly turbulent flow. Clair et al. [4] have presented a numerical and experimental investigation into the effect of sinusoidal leading edge serrations for the reduction of interaction broadband noise, generated due to high inflow turbulence level interacting with the leading edge. Reduction in sound power level over a wide frequency range between 3 and $4 \mathrm{~dB}$ were both simulated and measured. It was pointed out that the reason for the modest noise reductions, compared to the much larger noise reductions (up to $7 \mathrm{~dB}$ ) measured by Narayanan et al. [5], is due to the relatively short serration amplitude investigated. These results highlighted the importance of serration amplitude in the reduction of interaction broadband noise. This point was further demonstrated by Biedermann et al. [6] where a ranking of the influencing parameters clearly indicated that the serration amplitude contributes more than the serration wavelength in the reduction of interaction broadband noise.

While leading edge serrations have shown to be acoustically beneficial, the aerodynamic effects are controversial. Soderman [7] was one of the first to study the aerodynamic performance of biologically inspired wings. He found that leading edge serrations reduce the lift curve slope and increase maximum lift by 12 percent. Wei et al. [8] found that a counter-rotating vortex pairs were formed over each serration, which essentially changed the flow in the near surface region and mitigated the flow separation at post stall angles of attack. Johari et al. [9] and Hansen et al. [10] observed

\footnotetext{
*Assistant Professor, Faculty of Aerospace Engineering, oksana.s@ technion.ac.il, AIAA Member.

${ }^{\dagger}$ Senior Lecturer, Department of Mechanical and Aerospace Engineering, t.p.chong@brunel.ac.uk, AIAA Member.
} 
that the leading edge serrations could reduce the maximum lift coefficient in the pre-stall regime but increase the lift coefficient in the post-stall regime.

The problem of predicting the aerodynamic properties of any given arbitrary airfoil geometry with no restriction as to its thickness, camber, and angle of attack was addressed by Munk [11], Birinbaum[12], and Glauret [13]. Despite the fact that each airfoil has unique geometry the response to changes in angle of attack can be described by the classic inviscid thin airfoil theory [14-16]. In its framework, the classic thin airfoil theory predicts a slope of a lift coefficient $C_{L}$ of a thin wing in incompressible steady fluid as a function of an angle of attack $\alpha$ to be equal to $d C_{L} / d \alpha=2 \pi$. In practice, it is indeed common to measure the lift slope coefficient near the $d C_{L} / d \alpha=0.1 \pm 0.02 \mathrm{deg}^{-1}$ for the NACA 4 and 5 digits series. For example, NACA 0020 airfoil section exhibits a lift curve slope of $0.0899 \mathrm{deg}^{-1}[17]$.

The airfoil geometry is undoubtedly an important parameter affecting the aerodynamic performance. Until recently most of the work on aerodynamic performance of wings with leading edge serrations has focused on understanding flow characteristics of symmetric airfoil sections at high angles of attack, such as NACA 0012 [18], NACA 0020 [19, 20], NACA $0021[10,10,21,22]$. Only a handful of studies investigated the effect of leading edge serrations on the aerodynamic performance of asymmetric airfoil sections. One example where this is not the case is provided by Hansen et al. [10]. They concluded that leading edge tubercles are more beneficial for the asymmetric NACA 65-021 airfoil, compared to the NACA 0021 airfoil. For one particular cambered airfoil, NACA 65-(12)10, it has been shown in various studies to work well with leading edge serration on the interaction broadband noise reduction [4, 6, 23, 24]. However, to the best of our knowledge there are no studies that document aerodynamic performances especially for the 'cut-in' serration type [24]. This is particularly interesting since at zero angle of attack the cambered NACA 65(12)-10 airfoil section emits leading edge-turbulence interaction noise at a higher level when its acoustic signature is compared to the symmetric NACA 0012 airfoil section.

When discussing sinusoidal leading edge serrations, the wavelength $\lambda$ and the amplitude $a$ are often used to describe the modified geometry. It has been widely reported that within the linear range, the lift slope $d C_{L} / d \alpha$ at pre-stall angles of attack decreases with the increase of serration amplitude [9, 25]. The add on of serrations to the leading edge clearly has a potential to invalidate the classic thin airfoil theory assumtpions and results. Most importantly, it suggests a fundamental change in the lift-generation mechanism. However, it remains inconclusive of whether a new mechanism will exist because there are different approaches in the literatures when defining the planform area of the airfoil subjected to serrated leading edge. In the present work, the authors seek to discuss the definition of lift coefficient for a finite wing with leading edge serration in terms of the lift slope $d C_{L} / d \alpha$ produced at the pre-stall angles of attack.

\section{Experimental Setup}

The two dimensional cross-section of the airfoil under investigation here is a highly cambered NACA 65-(12)10 cascade compressor blade [26]. The NACA 65-(12)10 airfoil geometry is designed based on the NACA 65-(216)010 


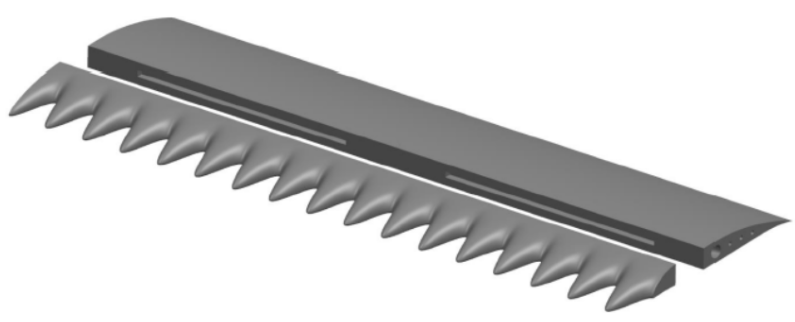

(a)

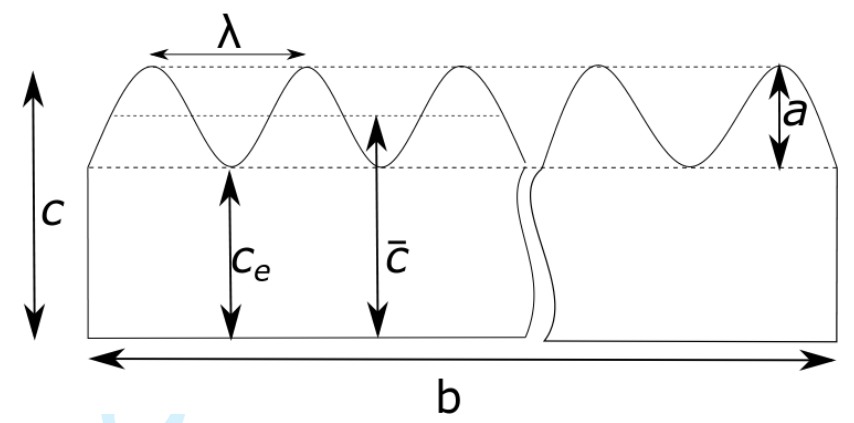

(b)

Fig. 1 (a) Illustrations of the the modified NACA 65(12)-10 airfoil geometry with the leading edge serrations and (b) the planform area geometry.

airfoil section by combining a basic thickness form with cambered mean line. The highly cambered airfoil has 5\% camber and 10\% thickness (see Figure 1a. The NACA 65 -series airfoil is a high performance airfoil designed to maintain laminar flow over a large portion of its upper surface [27]. Here 65 refers to the 'series', 12 is the 10 times the design lift coefficient at zero angle of attack and the last two digits refer to the blade relative thickness in percentage of the chord. The NACA 65-(12)10 airfoil section is asymmetric with the angle between the chord line and tangent to the lower surface of $1.4^{\circ}$ [28]. The NACA 65 airfoil series was used on aircraft building during World Word II, perhaps most noticeably on the P-51 'Mustang' fighter aircraft [27].

NACA 65-(12)10 airfoil geometry with leading and trailing edge serrations was previously studied by Liu et al. [29], Finez et al. [30], Vathylakis et al. [31], Gruber et al. [32], Clair et al. [4], Polacsek et al. [23], Biedermann et al. [6] and Chong et al. [24], to name a few. Within the scope of the current study, the sinusoidal leading edges were 'cut-in' directly into the main body of the wing with chord $c=0.15 \mathrm{~m}$, thus resulting in a mean chord $\bar{c}=c-a / 2$. The leading edge section was placed between $x / c=0$ and $x / c=0.33$; further downstream, $0.33<x / c<1.0$, was the unmodified airfoil. Once attached, the sinusoidal leading edge forms a continuous profile; thus giving the appearance that the sinusoidal patterns are 'cut-in' into the main body of the wing. Three tip to trough serration amplitudes, $a=7.5,30$, $45 \mathrm{~mm}$ were investigated (see Figure $1 \mathrm{~b}$ ), which result in the amplitude to mean chord ratio of $a / c=0.05,0.2$ and 0.3 , respectively. A similar ratio also applies to the wavelength $\lambda / c=0.1$ and 0.2 . Thus, a total 6 sinusoidal leading edge sections and one straight (baseline) leading edge were investigated.

Aerodynamic measurements were conducted in a closed test-section wind tunnel facility at Brunel University 
London, the UK. The wind tunnel has a rectangular test section of $0.5 \mathrm{~m} \times 0.5 \mathrm{~m}$, and the overall length of the test section is about $2.5 \mathrm{~m}$. The maximum velocity that can be reached is about $38 \mathrm{~ms}^{-1}$ and the freestream turbulence intensity is about $0.2-0.3 \%$ at this velocity. The airfoil models were mounted horizontally across almost the entire width of the test section, leaving $1 \mathrm{~mm}$ gap between the edge of the model and the test section walls. In order to investigate the effect of the sinusoidal leading edges on the aerodynamic performance, a 3-component strain gauge force balance was used to measure the aerodynamic forces. To allow accurate force and moment measurements, $1 \mathrm{~mm}$ gap between the airfoil and each side of the wind tunnel side window was facilitated, thus resulting in wing span of $b=0.498 \mathrm{~m}$. For clarity, the lift coefficient data is presented at a chord Reynolds number of $R e_{c}=\rho c U_{\infty} / \mu=1.5 \times 10^{5}$ where $\rho$ is the density, $U_{\infty}=15 \mathrm{~ms}^{-1}$ is the free-stream velocity, and $\mu$ is the dynamic viscosity of air. Only a single Reynolds number was chosen in our study because the aerodynamic performance is not expected to vary significantly across the speed range of the wind tunnel.

\section{Results}

In the description of the aerodynamic performance of a wing, the lift coefficient slope is one of the important parameters. The lift coefficient $C_{L}$ is defined as a ratio between the lift force $L$ and the product of dynamic pressure $q=0.5 \rho U_{\infty}^{2}$ and the wing planform area $S$. It is worth noting that when a wing with leading edge serrations is considered there is an ambiguity in the definition of the planform area.

One of the common definitions is based on the baseline planform area. It allows a direct comparison between the conventional rectangular geometry and the modified 'cut-in' serrated leading edge geometry. With this common definition, the same baseline planform area, as the one encountered with the conventional rectangular wing geometry, is considered. With this definition the lift coefficient takes the form

$$
C_{L}^{(i)}=\frac{L}{q S^{(i)}}
$$

where the baseline planform area $S^{(1)}=c b$ is used. When this trivial definition is considered it can be observed in Figure $2 \mathrm{k}$ that the serration amplitude is the leading parameter, while the wavelength has a minor effect. The introduction of leading edge serrations results in reduction in the lift coefficient due to the decrease in lift coefficient slope $d C_{L}^{(1)} / d \alpha$ with increase of serration amplitude. When this trivial definition is considered, the addition of leading edge serration is then perceived as detrimental in terms of generated lift.

The second definition is the most common in the relevant literature, based on the 'wetted' planform area. For a wing with sinusoidal leading edge the 'wetted' planform area $S^{(2)}=\bar{c} b$ is defined as a product between the mean chord $\bar{c}=c-a / 2$ and the span, which results in the same definition of lift coefficient,only with $S^{(2)}$. The effect of scaling of the lift coefficient by the mean chord is shown in Figure $2 \mathrm{p}$. Despite achieving slight improvement, the lift 


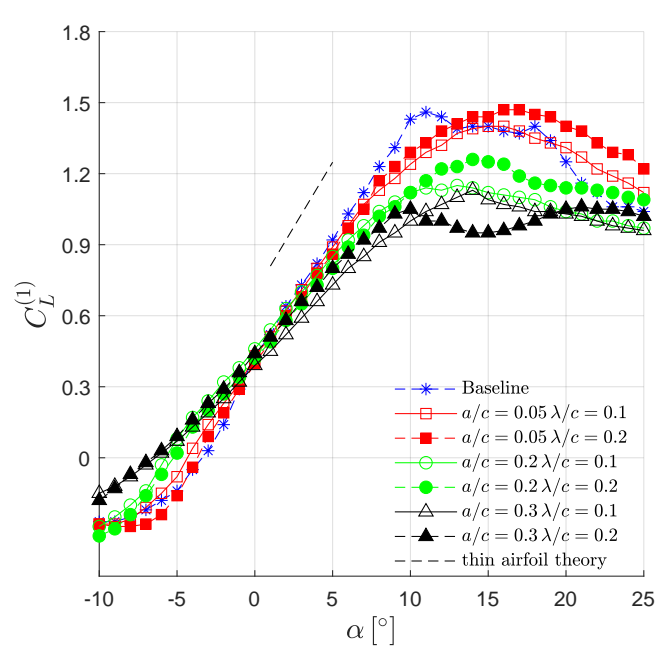

(a)

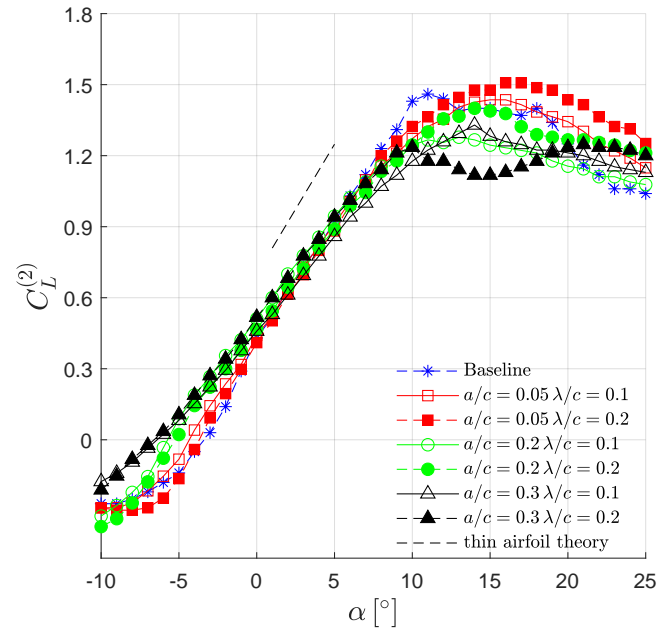

(b)

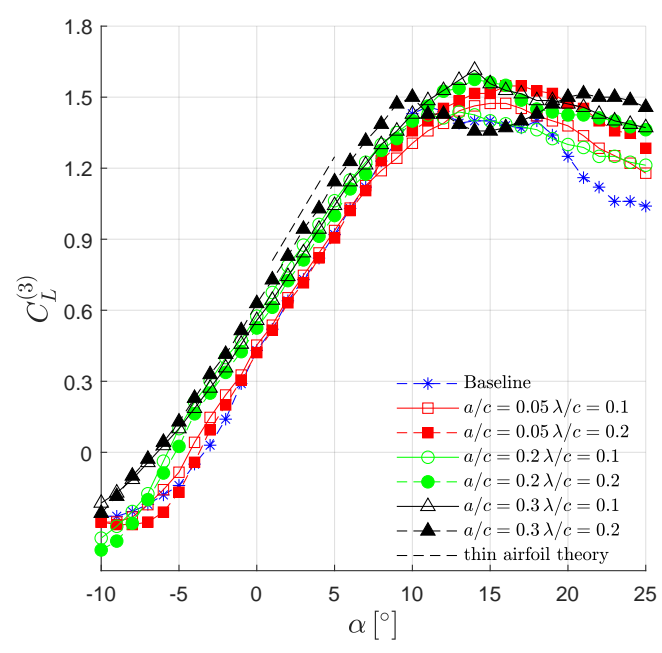

(c)

Fig. 2 Lift coefficient as a function of angle of attack at $R e_{c}=1.5 \times 10^{5}$, normalized with the (a) baseline chord $c$, (b) mean chord $\bar{c}$, (c) effective chord $c_{e}$. Dashed black line shows the ideal lift coefficient curve slope $\left(d C_{L} / d \alpha=2 \pi \mathbf{r a d}^{-1}\right)$ as predicted by the thin airfoil theory, for reference. 
curve slope is still lower then the one predicted by the theoretical lift coefficient slope of $d C_{L} / d \alpha=2 \pi$ per radian (or $d C_{L} / d \alpha=0.110$ per degree). Furthermore, it is still a function of the serration amplitude.

Currently, we propose an alternative definition for the planform area $S^{(3)}$, which is based on normalization of the lift coefficient with the effective planform area; thus, resulting in lift coefficient being defined in the same manner as in Eq.(1). Here the effective area $S^{(3)}=c_{e} b$ is defined as a product between the effective chord $c_{e}=c-a$ and a span $b$. Normalization with the effective chord is underpinned by the hypothesis that the leading edge sawteeth do not contribute significantly in the generation of lift. The newly defined lift coefficient is shown in Figure 2 , almost the entire set of serrated leading edges follows the pre-stall lift curve slope of the baseline configuration, which for the cambered NACA 65(12)-10 airfoil is equal to $d C_{L} / d \alpha=0.099$. The lift curves achieve similar gradient and remain parallel in the pre-stall range of angles of attack. The above results is in line with the expectation that flow passing the sawteeth could be so complex and three-dimensional that when the lift generated by the sawteeth is added to the airfoil, it will result in deviation from the thin airfoil theory for the lift curve slope.

Table 1 Lift slope for serrated airfoil section

\begin{tabular}{lcccc}
\hline & \multicolumn{2}{c}{ Undulation Parameters } & & \\
\cline { 2 - 3 }$a / c$ & $\lambda / c$ & $d C_{L}^{(1)} / d \alpha$ & $d C_{L}^{(2)} / d \alpha$ & $d C_{L}^{(3)} / d \alpha$ \\
\hline 0.05 & 0.1 & 0.092 & 0.095 & 0.097 \\
0.05 & 0.2 & 0.093 & 0.095 & 0.097 \\
0.2 & 0.1 & 0.073 & 0.081 & 0.092 \\
0.2 & 0.2 & 0.076 & 0.084 & 0.095 \\
0.3 & 0.1 & 0.068 & 0.008 & 0.096 \\
0.3 & 0.2 & 0.069 & 0.081 & 0.098 \\
\hline
\end{tabular}

The lift curve slope for the modified geometries are summarized in Table 1 . The lift curve slope was estimated in the linear region, i.e. at $1^{\circ} \leq \alpha \leq 7^{\circ}$. For the large serrations wavelength of $\lambda / c=0.2$, the lift curve slope reduces to $d C_{L}^{1} / d \alpha=0.093$ for the $a / c=0.05$ and to $d C_{L}^{1} / d \alpha=0.076$ for $a / c=0.2$. This trend in aerodynamic behavior is in agreement with previously published results of Johari et al. [9] who measured lift curve slope of $d C_{L} / d \alpha=0.081$ with $\lambda / c=0.5$ and $a / c=0.125$ with modified NACA 63-021 airfoil geometry.

To examine the boundary layer behavior with the introduction of leading edge serrations at the pre-stall condition, oil flow visualization techniques was applied on the suction side of the airfoil surface (see Figure 3 . The sawteeth at the leading edge represent highly three-dimensional geometry to generate spanwise pressure gradient. As a consequence, cross-flow can be induced around the leading edge region, resulting in the formation of counter-rotating streamwise vortices that will propagate downstream. These vortical structures are clearly evident in the figure, but the extents of which are also dependent on the serration amplitude. For the small serration amplitude $a / c=0.05$, the vortices cannot fully suppress the separation region near the trailing edge that is otherwise present for the baseline airfoil. On the other 


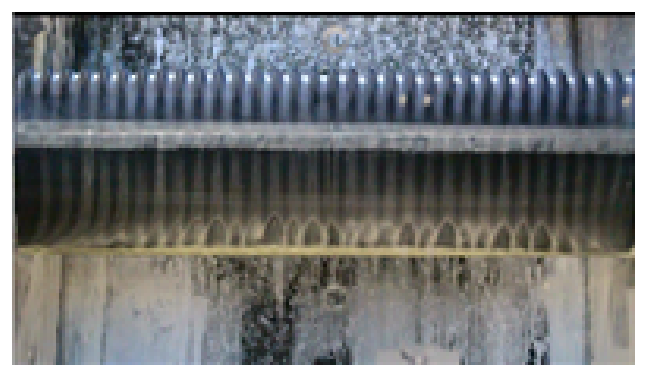

(a) $\lambda / c=0.1 \& a / c=0.05$

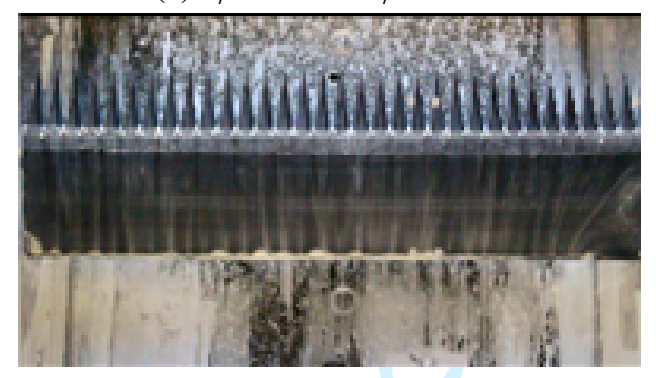

(c) $\lambda / c=0.1 \& a / c=0.3$

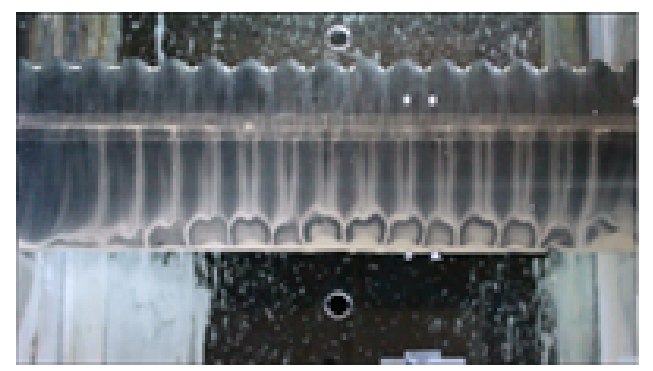

(b) $\lambda / c=0.2 \& a / c=0.05$

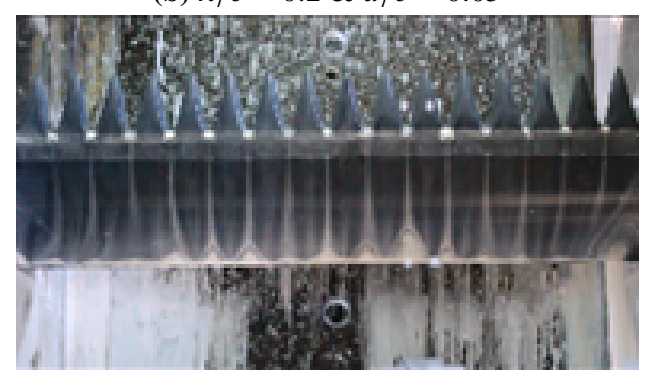

(d) $\lambda / c=0.2 \& a / c=0.3$

Fig. 3 Surface flow visualization on the suction side of NACA 65(12)-10 airfoil with leading-edge serrations at $\alpha=10^{\circ}$ angle of attack and $\operatorname{Re}_{c}=3.0 \times 10^{5}$.

hand, large serration amplitude $a / c=0.30$ seems to be capable of fully suppressing the trailing edge separation region. The above results suggest that stronger level of vortices are associated with larger serration amplitude.

\section{Conclusion}

One of the most important properties in wing design is how the lift coefficient varies with an angle of attack. The classic inviscid and incompressible thin airfoil theory predicts the lift coefficient slope with respect to angle of attack to be constant and equal to $d C_{L} / d \alpha=2 \pi$. The Reynolds numbers and thickness effects are not considered in this theory, and as a consequence it tends to over-predict the value of $d C_{L} / d \alpha$, unless there is a separation bubble. On the other hand, the viscous effects, which are also neglected by the theory, tend to decrease the value of $d C_{L} / d \alpha$. In practice, for most airfoil geometries at high Reynolds numbers the value of $d C_{L} / d \alpha$ obtained in wind tunnel experiments is a result of these counter-balancing effects, which brings most common airfoil performance remarkably close to the theory. At large angles of attack where the boundary layer tends to separate from the surface, the thickness of the boundary layer effectively distorts the geometry of the airfoil section. One outcome of this distortion is that the theoretical slope of the lift curve is no longer realized [14].

Within the scope of the current study we propose a simple correction to the definition of the planform area when leading edge serrations are introduced. Particularly, the manuscript discusses three definitions of the planfrom area and examines these definitions using a cambered NACA 65(12)-10 airfoil section with leading edge serrations, compared to the original baseline geometry. In line with previous findings, by introducing 'cut-in' serrated leading edge there is 
a reduction in the maximum lift coefficient and reduction of lift coefficient in pre-stall angles of attack when the lift coefficient is defined as a function of a conventional planform area, compared to the baseline airfoil with a straight leading edge. The reduction of lift coefficient at pre-stall angles of attack occurs due to the lower lift curve slope. However, when the effective chord is used to define the lift-generating area, there is a collapse in the lift curve slope. Most importantly, when the lift coefficient is normalized by the effective chord the effect of leading edge serrations is similar to the one observed with the baseline (straight) leading edge geometry.

The current work provides new insight into the aerodynamic mechanisms of airfoil behavior when subjected to serrated leading edges. We argue that for a serrated leading edge, spanwise pressure gradients are generated which in turn induce cross-flow around the leading edge region. As indicated by Serson et al. [18] the presence of the spawise pressure gradient for a serrated leading edge will distort and deflect the flow field significantly. Since the acceleration of the flow is responsible for generation of the suction peak near the airfoil's leading edge, the spanwise pressure gradient would hinder the ability to generate such suction peak. The aerodynamic effect of lower suction peak is similar to the one observed with lower angle of attack. Thus, when the lift coefficient is defined as a function of effective chord a collapse in the lift curve slope can be achieved. The purpose of the potential flow approach in the thin airfoil theory is to aid the analysis of the experimentally measured data by identifying the most significant lift generating mechanisms. Comparison with the ideal thin airfoil lift curve slope shows that the measured data approach this ideal lift coefficient slope.

\section{Acknowledgments}

The first author would like to acknowledge the support of Israel Science Foundation (individual grant 1702/16). The second author would like to thank Mr. Oliver Koster for his help in conducting part of the measurements.

\section{References}

[1] Graham, R., "The silent flight of owls," The Aeronautical Journal, Vol. 38, No. 286, 1934, pp. 837-843. doi:https: //doi.org/10.1017/S0368393100109915.

[2] Johnson, J. H., and Wolman, A. A., “The humpback whale, Megaptera novaeangliae.” Marine Fisheries Review, Vol. 46, No. 4, 1984, pp. 30-37.

[3] Fish, F., and Lauder, G., "Passive and active flow control by swimming fishes and mammals," Annu. Rev. Fluid Mech., Vol. 38, 2006, pp. 193-224. doi:https://doi.org/10.1146/annurev.fluid.38.050304.092201.

[4] Clair, V., Polacsek, C., Le Garrec, T., Reboul, G., Gruber, M., and Joseph, P., "Experimental and numerical investigation of turbulence-airfoil noise reduction using wavy edges," AIAA journal, Vol. 51, No. 11, 2013, pp. 2695-2713. doi: https://doi.org/10.2514/1.J052394. 
[5] Narayanan, S., Chaitanya, P., Haeri, S., Joseph, P., Kim, J., and Polacsek, C., “Airfoil noise reductions through leading edge serrations," Physics of Fluids, Vol. 27, No. 2, 2015, p. 025109. doi:https://doi.org/10.1063/1.4907798.

[6] Biedermann, T. M., Chong, T. P., Kameier, F., and Paschereit, C. O., "Statistical-Empirical Modeling of Airfoil Noise Subjected to Leading-Edge Serrations," AIAA Journal, 2017, pp. 3128-3142. doi:https://doi.org/10.2514/1.J055633.

[7] Soderman, P. T., "Aerodynamic effects of leading-edge serrations on a two-dimensional airfoil," NASA TM X-2643, 1972.

[8] Wei, Z., New, T., and Cui, Y., "An experimental study on flow separation control of hydrofoils with leading-edge tubercles at low Reynolds number," Ocean Engineering, Vol. 108, 2015, pp. 336-349.

[9] Johari, H., Henoch, C. W., Custodio, D., and Levshin, A., "Effects of leading-edge protuberances on airfoil performance," AIAA journal, Vol. 45, No. 11, 2007, pp. 2634-2642. doi:https://doi.org/10.2514/1.28497.

[10] Hansen, K. L., Kelso, R. M., and Dally, B. B., "Performance variations of leading-edge tubercles for distinct airfoil profiles," AIAA journal, Vol. 49, No. 1, 2011, pp. 185-194. doi:https://doi.org/10.2514/1.J050631.

[11] Munk, M. M., “General theory of thin wing sections,” NACA Report 142., 1922.

[12] Birnbaum, W., "Das ebene Problem des schlagenden Flügels," ZAMM-Journal of Applied Mathematics and Mechanics/Zeitschrift für Angewandte Mathematik und Mechanik, Vol. 4, No. 4, 1924, pp. 277-292.

[13] Glauert, H., The elements of aerofoil and airscrew theory, Cambridge University Press, 1983.

[14] Abbott, I. H., and Von Doenhoff, A. E., Theory of wing sections, including a summary of airfoil data, Courier Corporation, 1959.

[15] Karamcheti, K., Principles of ideal-fluid aerodynamics, Wiley New York, 1966.

[16] Ashley, H., Landahl, M., and Landahl, M. T., Aerodynamics of wings and bodies, Courier Corporation, 1965.

[17] Miklosovic, D., Murray, M., Howle, L., and Fish, F., "Leading-edge tubercles delay stall on humpback whale (Megaptera novaeangliae) flippers," Physics of fluids, Vol. 16, No. 5, 2004, pp. L39-L42. doi:https://doi.org/10.1063/1.1688341.

[18] Serson, D., Meneghini, J. R., and Sherwin, S. J., "Direct numerical simulations of the flow around wings with spanwise waviness," Journal of Fluid Mechanics, Vol. 826, 2017, pp. 714-731. doi:https://doi.org/10.1017/jfm.2017.475.

[19] Miklosovic, D. S., Murray, M. M., and Howle, L. E., "Experimental evaluation of sinusoidal leading edges," Journal of Aircraft, Vol. 44, No. 4, 2007, pp. 1404-1408. doi:https://doi.org/10.2514/1.30303.

[20] Favier, J., Pinelli, A., and Piomelli, U., "Control of the separated flow around an airfoil using a wavy leading edge inspired by humpback whale flippers," Comptes Rendus Mecanique, Vol. 340, No. 1-2, 2012, pp. 107-114. doi:https: //doi.org/10.1016/j.crme.2011.11.004. 
[21] Pérez-Torró, R., and Kim, J. W., “A large-eddy simulation on a deep-stalled aerofoil with a wavy leading edge,” Journal of Fluid Mechanics, Vol. 813, 2017, pp. 23-52. doi:https://doi.org/10.1017/jfm.2016.841.

[22] Hansen, K. L., Rostamzadeh, N., Kelso, R. M., and Dally, B. B., "Evolution of the streamwise vortices generated between leading edge tubercles," Journal of Fluid Mechanics, Vol. 788, 2016, pp. 730-766. doi:https://doi.org/10.1017/jfm.2015.611.

[23] Polacsek, C., Reboul, G., Clair, V., Le Garrec, T., and Deniau, H., “Turbulence-airfoil interaction noise reduction using wavy leading edge: An experimental and numerical study," Proc. of Inter-Noise, 2011.

[24] Chong, T. P., Vathylakis, A., McEwen, A., Kemsley, F., Muhammad, C., and Siddiqi, S., "Aeroacoustic and aerodynamic performances of an aerofoil subjected to sinusoidal leading edges," 21st AIAA/CEAS Aeroacoustics Conference, 2015 , pp. AIAA-2015-2200. doi:https://doi.org/10.2514/6.2015-2200.

[25] Zhang, M., Wang, G., and Xu, J., "Experimental study of flow separation control on a low-Re airfoil using leading-edge protuberance method," Experiments in fluids, Vol. 55, No. 4, 2014, p. 1710. doi:https://doi.org/10.1007/s00348-014-1710-z.

[26] Westphal, W. R., and Godwin, W. R., "Comparison of NACA 65-series Compressor-blade Pressure Distributions and Performance in a Rotor and in Cascade," NACA-TN-3806, 1957.

[27] Wright, T., and Gerhart, P., Fluid machinery: application, selection, and design, CRC press, 2009.

[28] Herrig, L. J., Emery, J. C., and Erwin, J. R., "Systematic two-dimensional cascade tests of NACA 65-series compressor blades at low speeds," NACA RM L51G31, 1957.

[29] Liu, X., Kamliya Jawahar, H., Azarpeyvand, M., and Theunissen, R., “Aerodynamic performance and wake development of airfoils with serrated trailing-edges," AIAA Journal, 2017, pp. 3669-3680. doi:https://doi.org/10.2514/1.J055817.

[30] Finez, A., Jacob, M., Jondeau, E., and Roger, M., "Broadband noise reduction with trailing edge brushes," 16th AIAA/CEAS aeroacoustics conference, 2010, p. 3980. doi:https://doi.org/10.2514/6.2010-3980.

[31] Vathylakis, A., Paruchuri, C. C., Chong, T. P., and Joseph, P., "Sensitivity of aerofoil self-noise reductions to serration flap angles," 22nd AIAA/CEAS Aeroacoustics Conference, 2016, p. 2837. doi:https://doi.org/10.2514/6.2016-2837.

[32] Gruber, M., Joseph, P., and Chong, T. P., “On the mechanisms of serrated airfoil trailing edge noise reduction,” 17th AIAA/CEAS aeroacoustics conference (32nd AIAA aeroacoustics conference), 2011, p. 2781. doi:https://doi.org/10.2514/6.2011-2781.

10

Submitted to AIAA Journal. Confidential - Do not distribute. 